\title{
Aplicación de ethephon en una población de mezquite (Prosopis spp.) para la extracción de goma en Naica, Chihuahua, México
}

\section{Application of ethephon in a mesquite population (Prosopis spp.) for gum extraction in Naica, Chihuahua, Mexico}

\author{
MAGAÑA-MAGAÑA， José Eduardo †’， SÁNCHEZ-BERNAL Jorge Alberto’， GONZÁLEZ- \\ ANCHONDO, María Elvira" y BARAY-GUERRERO, María del Rosario**
}

\begin{abstract}
Universidad Autónoma de Chihuahua, Facultad de Ciencias Agrícolas y Forestales, Delicias, Chihuahua, México.
"Universidad Autónoma de Chihuahua, Facultad de Contaduría y Administración, Delicias, Chihuahua, México.
\end{abstract}

ID $1^{\mathrm{er}}$ Autor: José Eduardo, Magaña-Magaña / ORC ID: 0000-0002-7582-1925, CVU CONACYT ID: 201541

ID $1^{\mathrm{er}}$ Coautor: Jorge Alberto, Sánchez-Bernal / ORC ID: 0000-0003-0282-0750, CVU CONACYT ID: 745559

ID $2^{\text {do }}$ Coautor: María Elvira, González-Anchondo / ORCID: 0000-0001-6111-1132 CVU CONACYT ID: 357321

ID $3^{\text {er }}$ Coautor: María del Rosario, Baray-Guerrero / ORC ID:0000-0003-4923-2278, CVU CONACYT ID: 732621

DOI: $10.35429 /$ JOTI.2020.13.4.30.40

Recibido 25 Enero, 2020; Aceptado 30 Junio, 2020

\section{Resumen}

El objetivo de la investigación fue extraer goma de la planta de mezquite, para ello, se utilizó una hormona vegetal en concentración al 10\%, 10.85, 15\%, 20\%, $25 \%$. Se aplicó en cortes de tronco y ramas secundarias de los árboles. Los resultados indicaron que el $100 \%$ de las plantas tratadas produjeron goma. La duración del efecto de la hormona en la producción de goma fue de nueve semanas. Las cosechas se realizaron cada tres semanas. Ninguna planta manifestó signos de daño o deterioro. Se observó que conforme el árbol tiene mayor perímetro de fuste produce mayor cantidad de goma. Se observó además que la hormona vegetal aparentemente no causó daño biológico en las hojas y en las vainas de acuerdo con la observación visual comparándolo con otros árboles sin tratamiento. El análisis químico proximal que ambas gomas de mezquite y huizache tienen semejanza e incluso mejoran los parámetros requeridos por la Food and Drug Administration para el uso de la goma arábiga en la industria alimenticia. Los periodos de producción y cosecha son de febrero a junio y de septiembre a diciembre. Se tiene una producción promedio estimado de alrededor de 180 gr por árbol temporada de producción.

Emulsificante, Industria alimenticia, Resinas, Especies nativas no maderables

\begin{abstract}
The objective of the research was to extract gum from the mesquite plant, for this, a plant hormone in concentration of $10 \%, 10.85,15 \%, 20 \%, 25 \%$ was used. It was applied to cuts of trunk and secondary branches of trees. The results indicated that $100 \%$ of the treated plants produced gum. The duration of the hormone's effect on gum production was nine weeks. Harvests were made every three weeks. No plant showed signs of damage or deterioration. It was observed that as the tree has a greater perimeter of the stem, it produces a greater amount of gum. It was also observed that the plant hormone apparently did not cause biological damage to the leaves and pods according to visual observation compared to other trees without treatment. The proximal chemical analysis that both mesquite and huizache gums have similarity and even improve the parameters required by the Food and Drug Administration for the use of gum arabic in the food industry. The production and harvest periods are from February to June and from September to December. There is an estimated average production of around $180 \mathrm{gr}$ per tree production season.
\end{abstract}

Emulsifier, Food industry, Resins, Non-timber native species

Citación: MAGAÑA-MAGAÑA, José Eduardo, SÁNCHEZ-BERNAL Jorge Alberto, GONZÁLEZ-ANCHONDO, María Elvira y BARAY-GUERRERO, María del Rosario. Aplicación de ethephon en una población de mezquite (Prosopis spp.) para la extracción de goma en Naica, Chihuahua, México. Revista de Invención Técnica. 2020. 4-13:30-40.

*Correspondencia al Autor (Correo Electrónico: rosariobaray@yahoo.com.mx)

$\dagger$ Investigador contribuyendo como primer autor. 


\section{Introducción}

El mezquite es considerado como un recurso valioso para comunidades rurales. Se distribuye como especie nativa en el sur de Estados Unidos de América (USA) y norte de México en zonas áridas y semiáridas. Se ha sobreexplotado principalmente como carbón y leña. En algunas comunidades es visto como agente invasor por lo que se han deforestado extensas áreas nativas de mezquite como una práctica sin control para usar las tierras en el cultivo y la ganadería. Por lo que se sugieren alternativas de manejo, aclareos y realizar plantaciones nuevas para seguir aprovechando sus recursos (CONAZA, 1994). El objetivo general de esta investigación es contribuir al desarrollo económico de los habitantes del desierto de Chihuahua de la región de Naica a través de la producción y cosecha de la goma de mezquite en áreas nativas y la preservación de esta especie. Se tomó el manejo de la goma en base a procedimiento usado por Reséndiz N. et al, (2016) en su estudio: goma de mezquite y huizache como alternativa de aprovechamiento en sistemas agroforestales y los sugeridos en el uso de esta en la elaboración de alimentos y su tratamiento.

Se persiguieron los siguientes resultados: Producir goma de mezquite en poblaciones nativas utilizando etileno en la localidad de Naica, Chihuahua, definir cuál técnica o tratamiento es mejor para la producción de la goma de mezquite sin que cause daño o deterioro al árbol, evaluar el comportamiento de las variables críticas de las poblaciones nativas como perímetro de fuste, altura de corte, diámetro de incisión de corte, grado de inclinación y profundidad, edad del árbol, producción de goma y verificar que no exista daño biológico o infestación de plagas, realizar un análisis proximal de la goma de mezquite al $15 \%$ y $20 \%$ y de huizache en laboratorio de fisicoquímica de la Facultad de Ciencias Químicas de la Universidad Autónoma de Chihuahua y compararlos con los resultados obtenidos por Reséndiz et al. (2016). E identificar las probables fuentes de compradores de la goma de mezquite teniendo como base el uso de la goma arábiga.

\section{Antecedentes}

Autoridades del ejido Naica, Chihuahua solicitaron una entrevista con autoridades e investigadores de la Facultad de Ciencias Agrícolas y Forestales (FCAyF) de la Universidad Autónoma de Chihuahua (UACH) para analizar la situación actual del ejido, en virtud de que la empresa dueña de la mina en su convenio de trabajo con el ejido le proporcionaba agua para irrigar 500 ha de cultivo. Al cerrar la mina en octubre de 2015, se dejó de contar con el agua. Así mismo manifestaron que tienen una superficie nativa de mezquite de aproximadamente 700 ha de diferentes edades. En la reunión con los ejidatarios se llegaron a los siguientes compromisos con la comunidad:

- Proyecto para la producción de goma de mezquite con apoyo de la FCAyF de la $\mathrm{UACH}$.

Procesar la vaina para uso de alimentación humana y animal.

Aprovechar la flor del mezquite en la alimentación de abejas para la producción de miel de abeja.

\section{Planteamiento del problema}

En virtud de que la empresa Peñoles, dueña de la mina en su convenio de trabajo con el ejido le proporcionaba agua para irrigar 500 ha de cultivo. Al cerrar la mina en octubre de 2015 , se dejó de contar con el agua, además de generar un gran desempleo.

\section{Justificación}

La FCAyF atendiendo el compromiso con el Ejido Naica, Saucillo y en su línea de investigación de generación y transferencia de tecnología apoya la investigación aplicada para la producción de goma de mezquite en dicho Ejido. Manifestaron que tienen una superficie nativa de mezquite de alrededor de 700 ha de diferentes edades. Se tiene una oportunidad de mercado para producir goma de mezquite debido a la demanda de la goma arábiga que es utilizada en la industria alimentaria, farmacéutica e industrial y es importada. 
Se establece el método de producción y cosecha y con este estudio se le dará al Ejido los resultados de su contenido bioquímico que permite establecer si es apto para el consumo humano y hacer el comparativo con anteriores estudios, además de buscar las fuentes para su manejo y purificación y el aprovechamiento comercial.

\section{Objetivos}

\section{General}

Investigar y aplicar el proceso de producir goma de mezquite bajo condiciones de poblaciones nativas utilizando una hormona vegetal en la localidad de Naica, Chihuahua.

\section{Específicos}

- Definir cuál técnica o tratamiento hormonal vegetal es mejor para la producción de la goma de mezquite sin que cause daño o deterioro al árbol.

Evaluar el comportamiento de las variables críticas de las poblaciones nativas como perímetro de fuste, altura de corte, longitud de incisión de corte, grado de inclinación y profundidad, edad del árbol, producción de goma y verificar que no exista daño biológico o infestación de plagas.

Realizar un análisis proximal de la goma de mezquite (Prosopis glandulosa) y compararlo con la de huizache (Acacia spp) y la goma arábiga.

Identificar las probables fuentes de compradores de la goma de mezquite teniendo como base el uso de la goma arábiga.

\section{Descripción del método}

El enfoque de este estudio es cuantitativo de tipo: exploratorio, descriptivo y explicativo. Se consideró el estudio realizado por Reséndiz N. et al. (2016), las sugerencias de los pobladores de la comunidad. Además, se inició un proceso de pruebas donde se definió la altura de los cortes, la longitud, la profundidad y el rango mínimo y máximo aceptable de la hormona vegetal sin que esta causara algún daño a la planta nativa de mezquite.

\section{Fuentes de información secundarias}

Tesis y artículos científicos y entrevistas con pobladores nativos.

\section{Fuentes de información primarias:}

\section{Localización del área de estudio}

La presente investigación se llevó a cabo en el Ejido Naica municipio de Saucillo; está situada en centro-sur del estado de Chihuahua tiene categoría de Sección Municipal del municipio de Saucillo. Sus coordenadas geográficas son $27^{\circ} 51^{\prime} 17^{\prime \prime} \mathrm{N} 105^{\circ} 29^{\prime} 33^{\prime \prime} \mathrm{O}$ y tiene una altitud de 1,340 metros sobre el nivel del mar, se localiza a 40 kilómetros al sur de la ciudad de Delicias y a 24 kilómetros al oeste de Conchos desde donde lo separan 10 kilómetros de la cabecera municipal, la ciudad de Saucillo.

Las principales vías de comunicación son dos carreteras estatales pavimentadas que la unen a Delicias y a Estación Conchos, en ambas poblaciones enlazando a la Carretera Federal 45 (Figura 1). Tiene 4,938 habitantes con 2492 hombres y 2446 mujeres. El clima es semiárido extremoso, con una temperatura máxima de $41.7^{\circ} \mathrm{C}$ y una mínima de $-14.1^{\circ} \mathrm{C}$; su temperatura media anual es de $18.3^{\circ} \mathrm{C}$. Tiene una precipitación pluvial media anual de 363.9 milímetros, con un promedio de 61 días de lluvia y una humedad relativa del $48 \%$; sus vientos dominantes son del sudoeste.

El mezquite es una planta nativa dentro la flora (INEGI 2010), representando la población de estudio. El suelo es propicio para actividades mineras, agrícolas y ganaderas. Hasta hace tres años la minería fue la principal fuente de empleo (80\%).

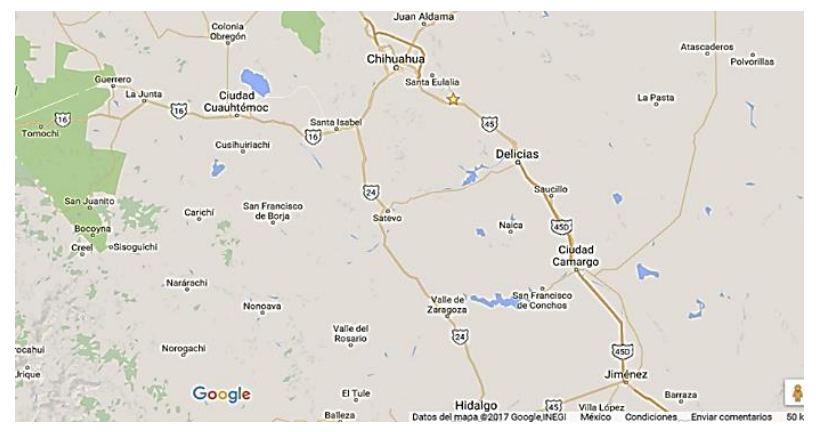

Figura 1 Macro localización 


\section{Población de estudio}

Área nativa de mezquite en el Ejido Naica, municipio de Saucillo, Chihuahua.

\section{Materiales, herramientas y equipo}

- Materiales: El producto a aplicar es una hormona vegetal (etileno) y agua destilada.

- Herramientas para limpiar y delimitar las áreas nativas: machetes, tijeras para poda, azadón, estacas y GPS.

Equipo para realizar los cortes en fuste y ramas: formón y martillo.

Equipo de aplicación: jeringas, recipientes para colocar la mezcla de la hormona vegetal con agua destilada, una para cada porcentaje de tratamiento.

Equipo de protección: guantes y máscara.

Equipo para recolección de la goma: formón, bolsas plásticas.

- Equipo para pesado: báscula de precisión.

\section{Metodología}

Se ubicaron las áreas de aplicación con el GPS para la toma de muestras.

Acondicionamiento de las áreas o cuadrantes a tratar para tener acceso a ellas debido a que son áreas densamente pobladas por el mezquite y otras especies realizando senderos con herramientas adecuadas para manejo y aclareo en vegetación.

Los tratamientos se aplicaron en cuadrantes de alrededor de $20 \mathrm{~m} \times 20 \mathrm{~m}$. Los tratamientos considerados para el estudio exploratorio de ver el rango de tratamientos que no cause daño y que exprese la producción de goma fueron: $10 \%, 15 \%, 20 \%$ y $25 \%$ para la producción de goma.
- $\quad$ Las mezclas se realizaron en contenedores de plástico o vidrio esterilizados uno por tratamiento (ver Tabla 1) y en cantidad requerida para el experimento a aplicar en el momento, debido a que una vez que se elabora la mezcla, el efecto de la hormona es de 6 horas.

\begin{tabular}{|r|r|r|}
\hline $\begin{array}{c}\text { Tratamiento } \\
\text { (Para100 ml) }\end{array}$ & \multicolumn{1}{c|}{ Etileno (ml) } & \multicolumn{1}{c|}{ Agua (ml) } \\
\hline $10.00 \%$ & 24 & 76 \\
\hline $10.85 \%$ & 26.04 & 73.96 \\
\hline $15.00 \%$ & 62.5 & 37.5 \\
\hline $20.00 \%$ & 83.33 & 16.66 \\
\hline $25.00 \%$ & 100 & 0 \\
\hline
\end{tabular}

Tabla 1 Composición de tratamientos por cada $100 \mathrm{ml} \mathrm{de}$ solución

Fuente: Elaboración propia

Se inició con una prueba el 15 de marzo de 2017 utilizando el tratamiento de $10.85 \%$, que fue aplicado a 5 árboles de mezquite para empezar el proceso de conocer el comportamiento del producto $\mathrm{y}$ su efecto en el árbol.

Una vez que se observó que no había daño, se hizo la segunda aplicación a 18 árboles con los tratamientos 10\%, 15\% y testigo el 28 de abril y recolectado el 3 de junio 2017 para determinar la altura en el tronco principal y la profundidad del corte.

- Con esta experiencia previa se aplicaron los tratamientos del $15 \%, 20 \%$ y $25 \%$. (el 21 de febrero de 2018 y recolectado en tres fechas).

- Se realizaron los cortes en tronco principal desde los $50 \mathrm{~cm}$ de altura del suelo y en 3 ramas secundarias con formón y martillo. El ángulo de corte fue de 30 a 35 grados. La longitud del corte dependió del perímetro del fuste y de la rama, que fue entre 2.5 y $5 \mathrm{~cm}$.

- Una vez atravesada la corteza, la profundidad del corte en el leño alrededor del cambium fue de medio a un centímetro, de acuerdo con el grosor de la corteza y del perímetro. 
Se aplicaron los tratamientos con una jeringa de $5 \mathrm{ml}$. La cantidad de mezcla de hormona vegetal más agua destilada aplicado a cada planta de mezquite nativa fue de la siguiente manera: un $\mathrm{ml}$ en el corte del tronco principal y $0.5 \mathrm{ml}$ en cada rama secundaria, usando equipo de protección: guantes y máscara.

La observación debe ser continua a las plantas tratadas y la recolección de la goma de mezquite se realizó en la tercera semana después de la aplicación del tratamiento en bolsas plásticas y se pesó en báscula de precisión; se continuó recolectando cada tres semanas hasta que dejó de escurrir.

Se realizó un análisis proximal a las muestras de la goma de mezquite al 15\% y $20 \%$ y huizache recolectadas en las áreas nativas por el laboratorio de análisis de agua y alimentos de la UACH (REG EF/573, S.S.A. REG JMA-PSAL-002-93. CED. PROF. 7915859), el 26 de junio de 2018.

\section{Resultados}

Para dar respuesta al objetivo 1: definir cuál técnica o tratamiento es mejor para la producción de la goma de mezquite sin que cause daño o deterioro al árbol, se presentan las siguientes tablas 2, 3, 4. El primer experimento exploratorio se realizó el 15 de marzo del 2017 a 5 árboles aplicándoles la hormona vegetal a base de etileno diluida con agua destilada y se visitó a la semana, observando que algunos árboles ya empezaban a escurrir la goma en los cortes principalmente, se prosiguió la observación semanalmente y se recolectó con formón en papel encerado, el 28 de abril y se pesaron en báscula de precisión en el laboratorio de suelos de la FCAyF. Escurrieron goma: el árbol 1= $50.48 \mathrm{gr}$, el árbol $2=48.61 \mathrm{gr}$, el árbol $3=21.84$ gr, el $4=9.8$ gr, el $5=29.35$ gr. Estos primeros indicios dieron la base para las siguientes aplicaciones. El primer experimento se realizó el 28 de abril, después de haber preparado con aclareos y senderos el área a tratar días antes a la aplicación con personal del Ejido asignado para dicha tarea. Estableciendo 18 árboles por área, 9 juveniles o de fuste delgado y 9 de fuste más grueso o adultos, a tres alturas con un mismo tratamiento del $10.85 \%$. (Tabla 2).

\begin{tabular}{|c|c|c|c|c|c|c|c|}
\hline \multirow[t]{2}{*}{$\begin{array}{c}\text { Altura } \\
\text { de } \\
\text { corte } \\
\text { cm }\end{array}$} & \multicolumn{2}{|c|}{$\begin{array}{l}\text { Tronco } \\
\text { Peso gr }\end{array}$} & \multicolumn{2}{|c|}{$\begin{array}{c}\text { Rama } \\
\text { secundaria } \\
\text { Peso gr }\end{array}$} & \multicolumn{2}{|c|}{$\begin{array}{l}\text { Asociado } \\
\text { Peso gr }\end{array}$} & \multirow[t]{2}{*}{ Total } \\
\hline & ST & CT & ST & CT & ST & CT & \\
\hline 50 & 0 & 16.23 & 0 & 9.28 & 0 & 4.73 & 30.23 \\
\hline 60 & 0 & 23.25 & 0 & 39.06 & 0 & 3.44 & 65.75 \\
\hline 70 & 1.55 & 7.49 & 0 & 14.98 & 0 & 3.74 & 27.76 \\
\hline
\end{tabular}

Tabla 2 Tratamiento al $10.85 \%$ de solución y tres alturas en el tronco principal. Aplicado el 28 de abril y recolectado el 3 de junio de 2017

Fuente: Elaboración propia con datos recolectados en campo

Como se observa en el cuadro 4 , los resultados indicaron que, de las tres alturas de corte en el tronco principal, la altura del corte de $60 \mathrm{~cm}$ en promedio fue mayor. El corte en rama secundaria produjo en promedio, un $34.7 \%$ más que la incisión de tronco. Pero de manera general el corte en el tronco principal por ergonomía se puede realizar desde los $50 \mathrm{~cm}$ de altura del piso en el tronco o fuste del árbol.

Al observar que en esta primera etapa la hormona vegetal no causó daño o problema a la planta, se procedió a elaborar otro experimento como segunda fase aplicado el 3 de mayo del 2017, usando como prueba los tratamientos del $10 \%, 15 \%$ y uno como testigo. A este último no se le aplicó la hormona vegetal, solo se le realizaron los cortes correspondientes: uno en el fuste desde una altura de los $50 \mathrm{~cm}$ del suelo con una longitud de aproximadamente $5 \mathrm{~cm}$ y los cortes en tres ramas a una distancia de la bifurcación de esta de alrededor de $15 \mathrm{~cm}$ a 30 cm según lo permitía el ángulo de inclinación de la rama con una longitud que oscilaba de 2.5 a 5 cm según el perímetro de esta.

Se aplicó un ml de solución de agua destilada con la hormona vegetal a base de etileno con una jeringa con aguja en el corte de fuste y $0.5 \mathrm{ml}$ en cada corte de cada rama secundaria, con una profundidad aproximada de $1 \mathrm{~cm}$ en esta fase. Se usó equipo de seguridad arriba mencionado. Se recolectó la goma de mezquite al mes de la aplicación de mismo con formón en papel encerado de cocina y se colocó en bolsitas individuales, una por árbol identificando cada una de ellas. Los resultados se observan en la Tabla 1. 


\begin{tabular}{|r|r|r|r|r|r|}
\hline \multicolumn{2}{|c}{ T1 (10\%) } & \multicolumn{3}{c|}{ T2 (15\%) } & \multicolumn{2}{c|}{$\begin{array}{c}\text { T3 (Sin } \\
\text { tratamiento) }\end{array}$} \\
\hline $\begin{array}{r}\text { Numero } \\
\text { de árbol }\end{array}$ & $\begin{array}{c}\text { goma } \\
\text { (gr.) }\end{array}$ & $\begin{array}{c}\text { Numero } \\
\text { de árbol }\end{array}$ & $\begin{array}{c}\text { goma } \\
\text { (gr.) }\end{array}$ & $\begin{array}{c}\text { Numero } \\
\text { de árbol }\end{array}$ & $\begin{array}{c}\text { goma } \\
\text { (gr.) }\end{array}$ \\
\hline 1 & 3.35 & 1 & 66.85 & 1 & 0 \\
\hline 2 & 0 & 2 & 22.15 & 2 & 0 \\
\hline 3 & 1.91 & 3 & 11.13 & 3 & 1.08 \\
\hline 4 & 0 & 4 & 17.67 & 4 & 0 \\
\hline 5 & 23.83 & 5 & 4.04 & 5 & 0 \\
\hline 6 & 47.36 & 6 & 39.67 & 6 & 0 \\
\hline Promedio & 12.74 & & 26.92 & & 0.18 \\
\hline
\end{tabular}

Tabla 3 Aplicación de tratamientos al 10\%, 15\% y sin tratamiento. Aplicado el 3 de mayo y recolectado en 3 de junio 2017

Fuente: Elaboración propia con datos recolectados en campo

Las observaciones que se desprenden de este cuadro son que los árboles con tratamientos del $15 \%$ y $10 \%$ escurrieron mayor cantidad de goma que los árboles a los que solo se les realizaron los cortes o incisiones. Así mismo se observa una amplia variabilidad entre los tratamientos del $10 \%$ y $15 \%$ y en promedio el tratamiento del $15 \%$ supera por encima del doble al del $10 \%$, pudiendo atribuírsele esto a la diversidad de variables que conlleva el experimento.

Se continuó con la siguiente fase experimental el 5 de julio del 2017 aplicando a dos áreas los tratamientos anteriores de lo que no se pudo conseguir información debido a que comenzó la época de lluvias y la goma se escurrió de los árboles debido a que la goma de mezquite es soluble en agua.

En el mes de octubre se aplicaron tratamientos a cuatro áreas que se nombraron de acuerdo a donde se ubicaban en el rodal tomando como referencia la característica que hacía posible encontrarlo con mayor facilidad, nombrándolas como: área de puerta, laguna de oxidación 1, laguna de oxidación 2 y el puente. En cada área se promediaron los pesos de la goma escurrida por tratamiento como se observa en la Tabla 4.

\begin{tabular}{|l|r|r|r|}
\hline Área & \multicolumn{1}{l}{$\begin{array}{l}\text { Promedio en } \\
\text { gr } \\
\text { Tratamiento } \\
\mathbf{1 0 \%}\end{array}$} & $\begin{array}{l}\text { Promedio en } \\
\text { gr }\end{array}$ & $\begin{array}{l}\text { Pratamiento } \\
\text { en gr S/T }\end{array}$ \\
\hline Puerta & 21.33 & 41.30 & 0.00 \\
\hline $\begin{array}{l}\text { Laguna de } \\
\text { Oxidación 1 }\end{array}$ & 26.10 & 22.88 & 1.50 \\
\hline $\begin{array}{l}\text { Laguna de } \\
\text { Oxidación 2 }\end{array}$ & 16.94 & 14.55 & 3.10 \\
\hline Puente & 22.76 & 30.11 & 0.50 \\
\hline Promedio/área & $\mathbf{2 1 . 7 8}$ & $\mathbf{2 7 . 2 1}$ & 1.28 \\
\hline
\end{tabular}

Tabla 4 Aplicación de tratamientos al 10\%, $15 \%$ y $\sin$ tratamiento el 14 de octubre y recolectado el 14 de noviembre de 2017

Fuente: Elaboración propia con datos recolectados en campo

Como se observa en el cuadro 6, continúa siendo el tratamiento del $15 \%$ el que más favorece al escurrimiento de goma, aunque se aprecia que en esta época de otoño - invierno es menor comparado con los aplicados al salir de la dormancia la planta que es en los primeros meses del año. También a lo largo de todo el año se observó escurrimiento en algunos árboles sin aplicación de etileno siendo más frecuente en el periodo de otoño - invierno, ya que hubo árboles llegaron a escurrir desde medio hasta más de un kilogramo de goma, los cuales fueron difíciles de cuantificar debido a que únicamente se recolectaban los que se encontraban cerca de las áreas de aplicación y en los árboles ubicados en los alrededores de la zona urbana y en los predios de cultivo.

En base a los resultados anteriores se procedió a aplicar nuevos tratamientos que fueron el $15 \%, 20 \%$ y $25 \%$ para observar el comportamiento de la planta conforme se aumenta el contenido de hormona, se muestran los resultados en la Tabla 5 .

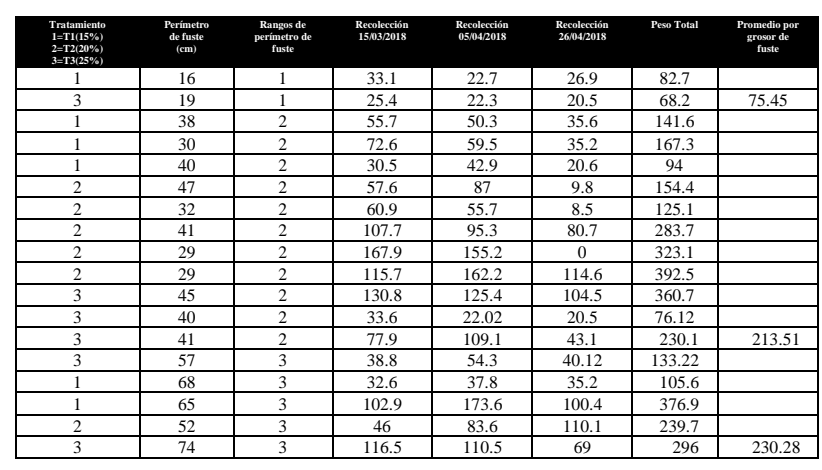

Tabla 5 Peso promedio en gr por árbol con tratamientos al $15 \%, 20 \%$ y $25 \%$ de solución. Aplicado el 21 de febrero de 2018 y recolectado en tres fechas. Pesos promedio por grosor de fuste

Fuente: Elaboración propia con datos obtenidos de la información recabada en campo 
Como se observa en la Tabla 5 existe, una amplia variación en los pesos totales de goma escurrida por árbol como respuesta a la aplicación de la hormona vegetal. Esto obedeció a la diversidad de los árboles que crecieron en las áreas nativas. Se concluyó que el árbol de mezquite produce goma controlada desde el perímetro de $15 \mathrm{~cm}$ como una respuesta a la hormona vegetal.

El problema que se presenta en estos árboles es la absorción del producto por eso las diferencias de producción de goma. A medida que los árboles tuvieron mayor perímetro la absorción de la hormona fue mejor. Y en general se observó mayor volumen de producción de goma. La altura de corte óptimo se obtuvo con árboles de un solo fuste, siendo este a partir de $50 \mathrm{~cm}$ del suelo para el tronco principal.

El árbol de mezquite tiene una característica de que a determinada altura del fuste principal (alrededor de un metro, metro y medio) se bifurca creando ramas secundarias con un promedio de tres.

La aplicación en las ramas secundarias fue a una distancia de la bifurcación entre 15 y $30 \mathrm{~cm}$ dependiendo del ángulo de la rama. La longitud del corte en tronco principal se estableció de $5 \mathrm{~cm}$ y para las ramas secundarias desde $2.5 \mathrm{~cm}$ a $5 \mathrm{~cm}$ dependiendo del grosor de esta. La inclinación del corte es de cerca de 30 a 35 grados.

La población nativa de mezquite compite en espacio y luz con distancia de un metro y medio entre arboles con diferentes edades creándose una especie de bosque de difícil acceso, para lo cual fue necesario el aclareo y la construcción de senderos. Considerando cuadrantes de aproximadamente de $20 \times 20 \mathrm{~m}$. Se seleccionaron 9 árboles jóvenes y 9 adultos de acuerdo con el grosor del tronco para ser tratados (total 18). Una vez atravesada la corteza, debido a que es la diferencia entre árboles jóvenes y adultos, la profundidad del corte en el leño alrededor del cambium fue de medio centímetro a un centímetro.
La observación debe ser continua a las plantas tratadas y la recolección de la goma de mezquite se realizó en la tercera semana después de la aplicación del tratamiento en bolsas plásticas y se pesó en báscula de precisión; se continuó recolectando cada tres semanas alrededor de 90 días hasta que dejó de escurrir. Para dar respuesta al objetivo 3 de esta investigación, se realizó el análisis proximal a las muestras de la goma de mezquite con los tratamientos del $15 \%$ y $20 \%$ recolectadas en las áreas nativas. Esta prueba fue realizada por el laboratorio de análisis de agua y alimentos de la UACH (REG EF/573, S.S.A. REG JMA-PSAL002-93. CED. PROF. 7915859), el 26 de junio de 2018. El análisis químico proximal mostro diferencias en el contenido de los carbohidratos y proteínas de ambas especies de acacias (Tabla 6). La goma de huizache presento el contenido menor de carbohidratos $(68.49 \%)$, este valor es aproximadamente un $20 \%$ menor que en la goma de mezquite al $15 \%(80.69 \%)$ y que en la goma de mezquite al $20 \%$ en comparación de la goma arábiga que se está tomando de referencia y no se reportó este dato.

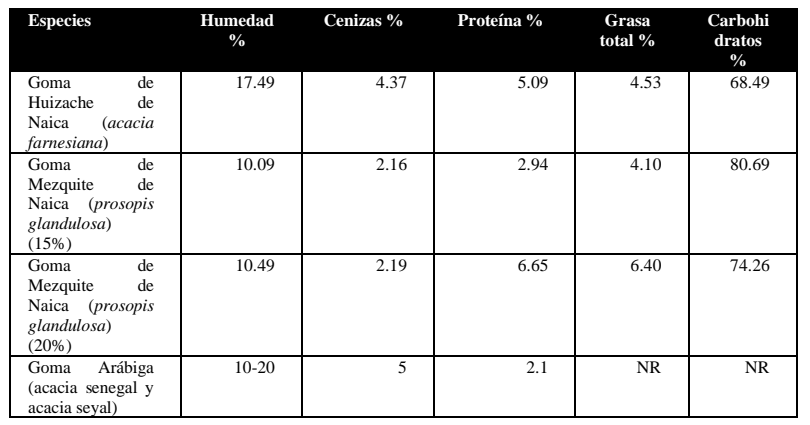

Tabla 6 Datos fisicoquímicos de las gomas analizadas comparadas con la goma arábiga

Fuente: Elaboración propia con datos de laboratorio de la Facultad de Ciencias Químicas de la UACH (Anexo 1) y Reséndiz et al. (2016)

Los carbohidratos son un importante componente químico que modifica la capacidad de espesamiento y promueve la estabilidad de emulsiones de agua (Adewusi et al., 2013). La fracción que presentan las gomas de carbohidratos juegan un papel muy importante en la estabilización, ya que esta fracción está asociada con el impedimento estérico entre las gotas de la emulsión, las cuales reducen y evitan la coalescencia de gotas por contacto (Gulrez, et al. 2011). 
Los altos contenidos de proteína están relacionados con la capacidad emulsionante. La goma de mezquite al (20\%) mostro el mayor contenido de proteína $(6.65 \%)$ que es 2 veces mayor que las otras gomas. Este valor es mayor que el contenido de proteína registrado para Paolii 0.34\% (Anderson \& Weiping, 1990). Así mismo, la acacia polyacantha tuvo un contenido de $2.9 \%$ (S. Mhinzi \& Mrosso.1995).

Algunos autores como Wang et al. (2011) menciona que las proteínas tienen una actividad superficial e interacciones electrostáticas que promueven la formación, engrosamiento y estabilización de la emulsión. Por lo que la proporción carbohidrato/proteína de la goma podría ser responsable de la estabilidad de las emulsiones.

La goma de huizache tuvo el contenido de ceniza mayor $(4.37 \%)$ que la goma de Mezquite al $15 \%(2.16 \%)$ a comparación con la referencia en donde estándares mínimos para la goma arábiga de buena calidad han sido definidos en la Farmacopea de los Estados Unidos, Edición XVII (1965) de la siguiente forma: $4 \%$ de cenizas totales (máximo), $0.5 \%$ de cenizas insolubles al ácido (máximo), $1 \%$ de residuo insoluble en agua (máximo).

Mientras que el contenido de humedad de la goma de Huizache fue mucho mayor $(17.49 \%)$ que las otras dos gomas. El contenido de grasas totales fue muy similar en todas las gomas analizadas.

Así mismo, se realizó la comparación de todos los parámetros del análisis proximal de las gomas de mezquite al $15 \%$ y $20 \%$ y huizache de las muestras recolectadas en Naica, Saucillo, Chihuahua con los resultados de Reséndiz, et al. (2016), como observan en la Tabla 7.

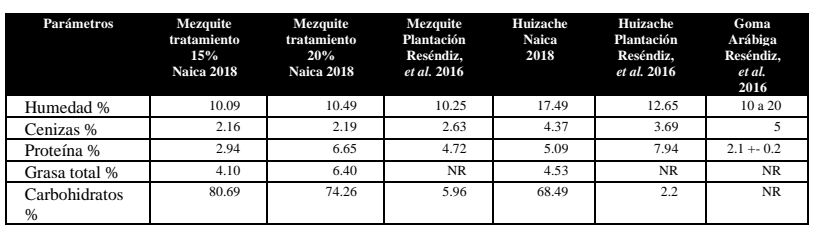

Tabla 7 Comparación de análisis proximal de la goma de mezquite con tratamiento aplicado al $15 \%$ y $20 \%$ y huizache en Naica Chihuahua (2018) versus resultados de Reséndiz, et al. (2016) en goma de mezquite, huizache y goma arábiga

Fuente: Elaboración propia con datos del laboratorio de análisis de agua y alimentos de la UACH (Anexo 1) y de Reséndiz, et al. (2016). NR = No reportado
Los resultados que se obtuvieron en el análisis proximal de las muestras de goma de mezquite obtenidas en Naica Chihuahua en el año 2018 se observan en el cuadro 6 y se compararon con los del estudio realizado en Celaya, Guanajuato por Reséndiz et al. (2016) en goma de mezquite, huizache y goma arábiga.

$\mathrm{Al}$ respecto las muestras de mezquite $\mathrm{y}$ huizache de Naica, Chihuahua dieron porcentajes de humedad muy similares a los obtenidos en Celaya, Guanajuato comparadas con la de la goma arábiga que observa un rango que va de $10 \%$ al $20 \%$, en dicho rango se encuentran todas las muestras en el entendido que en el límite inferior del $10 \%$ es el límite mínimo aceptable, en tanto el límite máximo de la goma arábiga que es el máximo aceptable. Esto es muy importante en virtud de que la humedad puede ser un factor con una ilusión óptica en el peso cuando su humedad es mayor.

El contenido de cenizas de la goma arábiga es del 5\%. Las muestras de la goma de mezquite de Naica, Chihuahua y Celaya, Guanajuato en relación con este parámetro, se encuentran en un rango de $2.16 \%$ a $2.63 \%$ que están por debajo del porcentaje de cenizas de la goma arábiga. En tanto que la goma de huizache de Naica, Chihuahua es la más cercano a la goma arábiga.

El contenido de proteína más alto fue el de la goma de huizache de Celaya, Guanajuato con $7.94 \%$. Siguiéndole en ese orden la goma de mezquite con el tratamiento del $20 \%$. Comparando los contenidos de proteína, encontramos que el porcentaje de la goma arábiga está por debajo de todos los porcentajes de las gomas de mezquite y huizache.

En el contenido de grasa total predomina el del mezquite con tratamiento al $20 \%$ y el huizache, obtenidas en Naica, Chihuahua. Los porcentajes de contenido de carbohidratos en mezquite y huizache de Naica, Chihuahua son mayores por encima de los obtenidos en las muestras de Celaya, Guanajuato sin poder compararse con la goma arábiga la cual no se reportó en este informe. 
Se puede concluir con los resultados de este análisis, que existen similitudes entre los porcentajes de los diferentes parámetros por lo tanto la goma de mezquite y la de huizache son productos sustitutos de la goma arábiga.

Así mismo dando respuesta al objetivo 4 , se establecieron las fuentes de compradores para su comercialización. Entre ellos se encuentran: BAFAR, Industrias Coca Cola, Dulces Montes, De la Rosa, Dulces Benny, Chocolates Ibarra, Cosméticos Natura, JAFRA, entre otros.

\section{Conclusiones}

Las gomas de mezquite y huizache son una alternativa para ofertarlas en el mercado nacional y frenar la importación de la goma arábiga (López-Franco et al, 2006).

Las resinas de Prosopis spp. (mezquite) y Acacia farnesiana (huizache) pueden sustituir a la goma arábiga que es usada como conservador natural de alimentos (Reséndiz et al., 2016).

- Se observó que la hormona vegetal aparentemente no causó daño biológico en las hojas y en las vainas de acuerdo con la observación visual comparándolo con otros árboles sin tratamiento.

Se comprobó mediante el análisis químico proximal que ambas gomas de mezquite y huizache tienen semejanza e incluso mejoran los parámetros requeridos por la Food and Drug Administration para el uso de la goma arábiga en la industria alimenticia.

Se definen dos periodos de producción y cosecha en meses de febrero a junio y de septiembre a diciembre.

Se tiene una producción promedio estimado de alrededor de 180 gr por árbol por temporada de producción.

\section{Recomendaciones}

Realizar el proceso de purificación de la goma para cubrir los estándares de calidad que demanda la industria y así competir con la goma arábiga.
- Realizar un experimento bajo condiciones de población nativa seleccionando plantas de mezquite con perímetro de fustes similares.

Seleccionar progenitores para reproducir los mejores productores de goma, a través de plantaciones respetando el hábitat.

- Mejorar la constitución fisiológica del árbol dándole espacios de cuatro metros entre planta y planta.

Una vez localizados los posibles compradores contactarlos e iniciar un proceso de negociación.

- Aprovechar integral y económicamente los recursos naturales considerando como el eje central su preservación y mejora.

Buscar financiamiento para la creación de un laboratorio propio que tenga la capacidad de purificar la goma.

\section{Referencias}

Acedo-Carrillo, J.I. \& Rosas-Durazo, A \& Herrera-Urbina, R \& Rinaudo, Marguerite \& Goycoolea, Francisco \& Valdez, Miguel. (2006). Zeta potential and drop growth of oil in water emulsions stabilized with mesquite gum. Carbohydrate Polymers. 65 . 327-336. 10.1016/j.carbpol.2006.01.016.

Adewusi, E.A., Founche, G., and Steenkamp, V. 2013. Effect of four medicinal plants on amyliod-beta induced neurotoxicity in $\mathrm{SH}-$ SY5Y. Cells. Afr. J Tradit Complement Altern. Med. 10: 6-11.

Anderson, D.M.W. \& Weiping, Wang. (1990). The characterization of Acacia paolii gum and four commercial Acacia gums from Kenya. Food Hydrocolloids. 3. 475-484. 10.1016/S0268-005X (09)80225-7.

Chávez, M. A. (2015). La vaina de mezquite (Prosopis spp) en la alimentación del ganado. Universidad Autónoma Agraria Antonio Narro, Torreón, Coahuila México. 
CONAZA (Comisión Nacional de las Zonas Áridas). 1994. Mezquite (Prosopis spp.). Cultivo alternativo para las zonas áridas y semiáridas de México. Instituto Nacional de Ecología. $30 \mathrm{p}$

Conferencia de la Naciones Unidas sobre comercio y desarrollo (2018). Comunicado de prensa: Goma arábiga: el aumento de la demanda crea nuevas oportunidades para los productores africanos. Ginebra, Suiza. https://unctad.org/es/Paginas/PressRelease.asp $\mathrm{x}$ ? OriginalVersionID $=451$

Gulrez, Syed \& Al-Assaf, Saphwan \& Phillips, Glyn. (2011). Hydrogels: Methods of Preparation, Characterisation and Applications. $10.5772 / 24553$.

Google maps. Disponible en: https://www.google.com/maps

Guía de productos químicos, 2015. Disponible en:

http://www.abellolinde.es/internet.lg.lg.esp/es/i mages/FDS-10021778-01-00-

ES316_89369.pdf?v=2.0

Instituto Nacional de Ecología (2007) Artículo: Descripción de la Planta. http://www2.inecc.gob.mx/publicaciones/libros /72/localiza.html

Investigación y Ciencia (2011). El etileno en la cocina. Disponible en: http://www.investigacionyciencia.es/blogs/fisic a-y-quimica/24/posts/etileno-en-la-cocina10362

López-Franco, Y., \& Goycoolea, F., \& Valdez, M., \& Calderón de la Barca, A. (2006). Goma de mezquite: una alternativa de uso industrial. Interciencia, 31 (3), 183-189.

López-Franco, Y. (2009). Uso del mezquite como fuente de polisacáridos de alto valor agregado. Centro de Investigación en Alimentación y Desarrollo, A. C. CONAFOR. Julio 2009.

Martínez Ruiz, A. (2011) El mezquite, árbol multipropósito. Artículo: Revista: El economista 2014. http:/eleconomista.com.mx/columnas/agronegocios/2011/04/26/mezquite-arbolmultiproposito
Meraz Vázquez, Salvador et.al. (1998). El mezquite, árbol de gran utilidad. Ciencias 51, julio-septiembre, 20-21.

Musa, H. H., Ahmed, A. A., \& Musa, T. H. (2017). Chemistry, Biological, and Pharmacological Properties of Gum Arabic. Reference Series in Phytochemistry, 118.doi:10.1007/978-3-319-54528-8_11-1

Organización de las Naciones Unidas para la Alimentación y la Agricultura (FAO). 2011. Situación de los bosques del mundo. Roma, Italia. $716 \mathrm{p}$.

Quiroz C., J.A. y M.A. Magaña A. 2015. Resinas naturales de especies vegetales mexicanas: usos actuales y potenciales. Madera y Bosques 21(3):171-183.

Reséndiz N. et al. (2016) Goma de mezquite y huizache como alternativa de aprovechamiento en sistemas agroforestales. Revista Mexicana de Ciencias Agrícolas, núm. 16, mayo-junio, 2016, pp. 3251-3261 Instituto Nacional de Investigaciones Forestales, Agrícolas y Pecuarias. Estado de México, México

Rodríguez Sauceda, Elvia Nereyda; Rojo Martínez, Gustavo Enrique; Ramírez Valverde, Benito; Martínez Ruiz, Rosa; Cong Hermida, Milagros de la Caridad; Medina Torres, Salvador Martín; Piña Ruiz, Hugo Humberto (2014). Análisis Técnico del Árbol del Mezquite (Prosopis laevigata Humb. \& Bonpl. ex Willd.) en México. Ra Ximhai, vol. 10, núm. 3, pp. 173193. Universidad Autónoma Indígena de México El Fuerte, México. Disponible en: http://www.redalyc.org/articulo.oa?id=461311 11013.

Román P., Horacio (2016). Bromatología de la Vaina de Mezquite (Prosopis spp.) Como Altrnatova de Consumo Sustentable en la Comarca Lagunera, Universidad Autónoma Agraria Antonio Narro. Torreón Cohauila, México.

S. Mhinzi, G \& Mrosso, Hillary. (1995). Studies on Tanzanian Acacia gums. Part 3. Some properties of gum exudates from the series Vulgares and Gummiferae. Food Chemistry FOOD CHEM. 54. 261-264. 10.1016/03088146(95)00038-K. 
Solís G., G. 1997. Evaluación poblacional actual del mezquite y palo fierro en ambientes áridos sujetos a un aprovechamiento continuo. CONACYT. 3888-N9401. Informe Final de Proyecto. Hermosillo, Sonora. 86 p.

Sosa Pérez, G. (2010). Tecnologías para el manejo integral de los bosques de mezquite en el norte de México. Instituto Nacional de Investigaciones Forestales Agrícolas y Pecuarias, apoyado por el fondo sectorial CONAFOR-CONACYT.

http://ford.ciesas.edu.mx/downloads/2do_2_01. pdf

Valenzuela, N. L. M., C. R. Trucios, S. J. C. Ríos, H. A. Flores, B. J. L. González. 2011. Caracterización dasométrica y delimitación de rodales de mezquite (Prosopis spp) en el estado de Coahuila. Revista Chapingo serie Ciencias Forestales y del Ambiente 17: 87-96.

Wang L, et al. (2011) Growth propagation of yeast in linear arrays of microfluidic chambers over many generations. Biomicrofluidics 5(4):44118-441189 